\title{
WHEN DOES UNIQUE LOCAL SUPPORT ENSURE CONVEXITY?
}

\author{
DONALD FRANCIS YOUNG
}

\begin{abstract}
A basic theorem of convex analysis states that a real-valued function on an open interval of the real line is convex and differentiable if at each point of its domain there exists a unique supporting line. In this paper we show that the same conclusion can be drawn under the weaker hypothesis that there exists a unique locally supporting line at each point. We also show by counterexample that convexity cannot be concluded under analogous circumstances for $f: S \rightarrow$ $\mathbb{R}$, where $S \subset \mathbb{R}^{n}$ is open and convex, if $n>1$.
\end{abstract}

\section{INTRODUCTION}

A basic theorem in the theory of convex functions states that if $f$ is a realvalued function on an open interval $I \subset \mathbb{R}$ and if $f$ has a unique supporting line at every $x \in I$, then $f$ is convex and differentiable [3, p. 12]. The main question addressed in the present article is whether this conclusion of convexity can be drawn under the weaker hypothesis that $f: I \rightarrow \mathbb{R}$ has unique local support at every point. We also consider the same question for the case of a function $f: S \rightarrow \mathbb{R}$, where $S$ is an open convex subset of $\mathbb{R}^{n}$. If there are no a priori assumptions (such as continuity), the answer turns out to be affirmative in the first instance but not in the second (for $n>1$ ). The consideration which prompts us to ask this question is the comparison with the well-known fact that a differentiable function $f: I \rightarrow \mathbb{R}$ can be proved convex with just the hypothesis of local support at each point.

In accordance with the usual definition, we say that $f: S \rightarrow \mathbb{R}$ has support at $x_{0} \in S$ if there exists an affine function $g: \mathbb{R}^{n} \rightarrow \mathbb{R}$ such that $f\left(x_{0}\right)=g\left(x_{0}\right)$ and $f(x) \geq g(x)$ for all $x \in S$; and when this condition holds we refer to the hyperplane $H=\left\{(x, g(x)) \mid x \in \mathbb{R}^{n}\right\}$ as a supporting hyperplane for $f$ at $x_{0}$. In a similar way, let us say that $f$ has local support at $x_{0}$ if $f\left(x_{0}\right)=g\left(x_{0}\right)$ and $f(x) \geq g(x)$ for all $x$ in some neighborhood of $x_{0}$, and let us refer to $H$ as a locally supporting hyperplane in this case. (If $n=1$, we shall naturally substitute the word line for hyperplane.)

In $[1$, p. 50] it is proved that for an upper semicontinuous function $f: I \rightarrow \mathbb{R}$ to be convex it is necessary and sufficient that the following condition hold for each $x_{0} \in I$ : if there exists an affine function $g$ such that $f\left(x_{0}\right)=g\left(x_{0}\right)$ and

Received by the editors October 26,1993; originally communicated to the Proceedings of the $A M S$ by Andrew Bruckner.

1991 Mathematics Subject Classification. Primary 26A51; Secondary 26A15, 26A21, 26A24, $26 \mathrm{~B} 25$.

Key words and phrases. Convex function, supporting line, Hausdorff Maximality Theorem. 
$f(x) \leq g(x)$ for all $x$ in some neighborhood of $x_{0}$, then the graphs of $f$ and $g$ coincide in some neighborhood of $x_{0}$. Using this fact, it is easy to prove the following. (See also [5].)

Theorem 1. Let $I$ be a nonempty open interval of $\mathbb{R}$, let $f: I \rightarrow \mathbb{R}$ be continuous, and suppose that at each $x \in I$ there exists a locally supporting line for $f$. Then $f$ is convex, and each of the locally supporting lines is actually a supporting line

If we also assume that each of the locally supporting lines in the hypothesis of this theorem is unique, then we can easily conclude that $f$ will have a unique supporting line at each $x \in I$, making $f$ differentiable as well as convex. Based on the result in [1], we actually could have stated Theorem 1 with the weaker assumption that $f$ was only upper semicontinuous. However, once we include the assumption of uniqueness, we can dispense with the assumption of continuity altogether. The main result which we shall prove is the following.

Theorem 2. Let $I$ be a nonempty open interval of $\mathbb{R}$, and suppose that $f: I \rightarrow \mathbb{R}$ has a unique locally supporting line at each $x \in I$. Then $f$ is convex.

Once this has been proved, differentiability follows immediately from Theorem 1, as stated above, since a convex function on an open interval is continuous. Without the assumption of uniqueness, Theorem 2 would be false, as illustrated by the counterexample $f(x)=-\llbracket x \rrbracket$ (where $\llbracket \cdot \rrbracket$ denotes the greatest integer function).

The idea of relaxing a priori assumptions of continuity in convexity results seems not to have come up often in the literature, but two recent papers which do take this approach are [2] and [4]. In [4], assuming only that $f: I \rightarrow \mathbb{R}$ is Darboux and of Baire class one (a weaker assumption than continuity), Weil proves that if the second lower symmetric derivate

$$
L D_{2}(x)=\liminf _{h \rightarrow 0^{+}}(f(x+h)+f(x-h)-2 f(x)) / h^{2}
$$

is nonnegative throughout $I$, then $f$ is convex. It is easy to see from this result that the assumption of continuity could correspondingly be relaxed to Darboux and Baire class one in Theorem 1 above, since the existence of a locally supporting line for $f$ at $x$ implies the nonnegativity of $L D_{2}(x)$. In [2], a general criterion is given for determining when it is possible to replace a hypothesis of continuity with a weaker hypothesis of Darboux and Baire class one in establishing that a given function-theoretic property will imply convexity.

\section{CONVEXITY VIA LOCALLY SUPPORTING LINES}

The proof of Theorem 2 is by contradiction, and the crucial step involves considering what happens when a locally supporting line fails to be actually supporting. This is the significance of Lemma 1 below.

Here and throughout the remainder of this section $f$ is a real-valued function on the open interval $I$. Since we shall be working in $\mathbb{R}^{2}$, let us adopt the commonly used terminology that a point $(x, y) \in \mathbb{R}^{2}$ lies above, on, or below the line $\{(\tilde{x}, g(\tilde{x})) \mid \tilde{x} \in \mathbb{R}\}$ (where $g: \mathbb{R} \rightarrow \mathbb{R}$ is affine) accordingly as $y>g(x)$, $y=g(x)$, or $y<g(x)$. Similarly, we say that $(x, y)$ lies above (or on or below) the line segment $\{(\tilde{x}, g(\tilde{x})) \mid a \leq \tilde{x} \leq b\}$ if $a \leq x \leq b$ and $y>g(x)$ (or, respectively, $y=g(x), y<g(x)$ ). 
Lemma 1. Let $a, b \in I$ with $a<b$, and suppose that $f$ has $a$ unique locally supporting line $L_{a}$ at $a$ and a unique locally supporting line $L_{b}$ at $b$. Then

(a) If $S=\left\{\tilde{b} \mid \tilde{b}>a\right.$ and $(\tilde{b}, f(\tilde{b}))$ lies below $\left.L_{a}\right\}$ is nonempty and $b=\inf S$, then $Q=(b, f(b))$ lies on $L_{a}$.

Likewise,

(b) If $T=\left\{\tilde{a} \mid \tilde{a}<b\right.$ and $(\tilde{a}, f(\tilde{a}))$ lies below $\left.L_{b}\right\}$ is nonempty and $a=$ $\sup T$, then $P=(a, f(a))$ lies on $L_{b}$.

Proof. To prove assertion (a), suppose that $S$ is nonempty and $b=\inf S$. For all $\tilde{b}<b$ sufficiently near $b,(\tilde{b}, f(\tilde{b}))$ lies on or above $L_{a}$. Thus, if $Q$ lay below $L_{a}$ there would exist a $y>f(b)$ such that for all $\tilde{b}<b$ sufficiently near $b, f(\tilde{b})>y$. This would imply that every line through $Q$ with slope less than or equal to the slope of $L_{b}$ would be a locally supporting line for $f$ at $b$, contradicting the uniqueness of $L_{b}$. On the other hand, for all $\tilde{b}>b$ sufficiently near $b,(\tilde{b}, f(\tilde{b}))$ lies on or above $L_{b}$. Thus, if $Q$ lay above $L_{a}$, then $(\tilde{b}, f(\tilde{b}))$ would lie above $L_{a}$ for all $\tilde{b}>b$ sufficiently near $b$, contradicting the assumption that $b=\inf S$. Therefore, $Q$ must lie on $L_{a}$.

Assertion (b) is proved similarly.

In the situation described in the first assertion of Lemma 1, note that there will exist numbers $\tilde{b}>b$ arbitrarily close to $b$ with $(\tilde{b}, f(\tilde{b}))$ lying below $L_{a}$. Since $Q$ lies on $L_{a}$, it follows that the slope of $L_{b}$ will be less than the slope of $L_{a}$, so the point $(a, f(a))$ must lie below $L_{b}$ and $\{\tilde{a} \mid \tilde{a}<b$ and $(\tilde{a}, f(\tilde{a}))$ lies below $\left.L_{b}\right\}$ must be nonempty. If $a^{\prime}$ denotes the supremum of this set, then $a^{\prime}<b$ since $L_{b}$ is a locally supporting line for $f$ at $b$. Furthermore, the interval $[a, b]$ will satisfy the following two important properties:

(P1) For all $x \in[a, b]$, the point $(x, f(x))$ lies above or on the closed line segment $L[a, b]$ connecting the points $P=(a, f(a))$ and $Q=$ $(b, f(b))$.

(P2) The line through the points $P=(a, f(a))$ and $Q=(b, f(b))$ either fails to be a locally supporting line for $f$ at $a$ or fails to be a locally supporting line for $f$ at $b$.

If $f$ has a unique locally supporting line $L_{a^{\prime}}$ at $a^{\prime}$ also, then $\left[a^{\prime}, b\right]$ also will satisfy these two properties, by part (b) of Lemma 1 . Note that we define $L[a, b]=\{(x, g(x)) \mid a \leq x \leq b\}$, where $g$ is the affine function $g(x)=$ $f(a)+(f(b)-f(a))(x-a) /(b-a)$. Together, (P1) and (P2) say that $f(x) \geq g(x)$ for $a \leq x \leq b$ but for every $\varepsilon>0$ there exists an $x \in(a-\varepsilon, b+\varepsilon)$ such that $f(x)<g(x)$.

Let us denote by $\mathscr{F}_{f}$ the family of all closed intervals $[a, b] \subset I$ such that $a<b$ and (P1) and (P2) hold. A necessary and sufficient condition for $f: I \rightarrow \mathbb{R}$ to be convex is that at each $x \in I$ there exists a supporting line for $f$ [3, p. 12]. Thus, if $f$ satisfied the hypothesis of Theorem 2 but were not convex, one could apply Lemma 1 inductively, alternating between parts (a) and (b) as indicated immediately following the proof of Lemma 1 , to generate a nested sequence of intervals $\left\{\left[a_{i}, b_{i}\right]\right\}_{i=1}^{\infty}$ with $\left[a_{i}, b_{i}\right] \in \mathscr{F}_{f}$ for all $i$. According to Lemma 3 below, if the intersection of these intervals is not just a single point, then this intersection will also belong to $\mathscr{F}_{f}$.

In proving Theorem 2, we will apply the Hausdorff Maximality Theorem to the family $\mathscr{F}_{f}$. What turns out to be important there is not the particular 
method by which the nested sequence $\left\{\left[a_{i}, b_{i}\right]\right\}$ is generated but rather that each of the intervals satisfies the properties $(\mathrm{P} 1)$ and $(\mathrm{P} 2)$.

Lemma 2. Let $a \in I$, let $\left\{a_{n}\right\}$ be a sequence in I converging to $a$, and suppose that there exists a locally supporting line $L$ for $f$ at $a$. Then $\liminf _{n \rightarrow \infty} f\left(a_{n}\right)$ $\geq f(a)$.

Proof. It follows easily from the existence of $L$ that for every $\varepsilon>0$ there exists a positive integer $N_{\varepsilon}$ such that for all $n \geq N_{\varepsilon}, f\left(a_{n}\right)>f(a)-\varepsilon$.

Note that Lemma 2 implies that a function which satisfies the hypothesis of Theorem 2 must be lower semicontinuous.

Lemma 3. Let $\left\{\left[a_{i}, b_{i}\right]\right\}_{i=1}^{\infty}$ be a sequence of closed subintervals of $I$, with $\left[a_{j}, b_{j}\right] \subset\left[a_{i}, b_{i}\right]$ for $j>i$ and with $\lim _{i \rightarrow \infty} a_{i}<\lim _{i \rightarrow \infty} b_{i}$, and suppose that $\left[a_{i}, b_{i}\right] \in \mathscr{F}_{f}$ for all $i$. Suppose also that there exist locally supporting lines for $f$ at $a=\lim _{i \rightarrow \infty} a_{i}$ and at $b=\lim _{i \rightarrow \infty} b_{i}$. Then $[a, b] \in \mathscr{F}_{f}$.

Proof. Denote each point $\left(a_{i}, f\left(a_{i}\right)\right)$ by $P_{i}$ and each point $\left(b_{i}, f\left(b_{i}\right)\right)$ by $Q_{i}$. We claim that $\lim _{i \rightarrow \infty} P_{i}=P$ and $\lim _{i \rightarrow \infty} Q_{i}=Q$, where $P=(a, f(a))$ and $Q=(b, f(b))$. By property (P1), $f$ is bounded below on $\left[a_{1}, b_{1}\right]$.

The claim that $\lim _{i \rightarrow \infty} P_{i}=P$ is obvious in the case $a_{i}=a$ for some $i=n$, for then $a_{i}=a$ for all $i \geq n$. Thus, let us consider the case in which $a_{i}<a$ for all $i$. Since $(\mathrm{P} 1)$ holds for $\left[a_{i}, b_{i}\right], P$ cannot lie below $L\left[a_{i}, b_{i}\right]$, and so we must have

$$
f\left(b_{i}\right) \leq \frac{f(a)-f\left(a_{i}\right)}{a-a_{i}}(b-a)+f(a)
$$

whenever $f\left(a_{i}\right)>f(a)$. Since the numbers $f\left(b_{i}\right)$ are bounded below, it follows that $\lim \sup _{i \rightarrow \infty} f\left(a_{i}\right) \leq f(a)$. Combining this result with the result of Lemma 2 , we see that $\lim _{i \rightarrow \infty} f\left(a_{i}\right)$ exists and equals $f(a)$. Thus, $P_{i} \rightarrow P$. That $Q_{i} \rightarrow Q$ is proved similarly. Using these two facts, we easily deduce that, since (P1) holds for each interval $\left[a_{i}, b_{i}\right],(\mathrm{P} 1)$ holds for $[a, b]$ as well.

Now we must establish that property (P2) holds for $[a, b]$. If $a_{i}=a$ for some $i=n$ and $b_{i}=b$ for some $i=m$, then for all $i \geq \max \{n, m\},[a, b]=$ $\left[a_{i}, b_{i}\right]$. In this case (P2) holds for $[a, b]$ because it holds for all the intervals $\left[a_{i}, b_{i}\right]$. Hence, we need to establish (P2) for the case in which $a_{i} \neq a$ for all $i$ and also for the case in which $b_{i} \neq b$ for all $i$. The argument is essentially the same in both cases, however, so let us proceed under the presumption that $a_{i} \neq a$ for all $i$. We shall examine four subcases, which are not mutually exclusive but which exhaust all possibilities. Denote the line through the points $P$ and $Q$ by $L$.

Case 1: $P_{i}$ lies below $L$ for infinitely many $i$. In this case, since $P_{i} \rightarrow P$, given any $\varepsilon>0$ there will exist infinitely many $i$ such that $P_{i}$ lies below $L$ and $a-a_{i}<\varepsilon$. Thus, $L$ is not a locally supporting line for $f$ at $a$, so (P2) holds for $[a, b]$ in this case.

Case 2: $Q_{i}$ lies below $L$ for infinitely many $i$. The same kind of argument as in Case 1 shows here that $L$ is not a locally supporting line for $f$ at $b$. Again, (P2) holds for $[a, b]$.

Case 3: $P_{i}$ lies above $L$ for infinitely many $i$. In this case, for any $i$ such that $P_{i}$ lies above $L$, the point $Q_{i}$ lies below $L$, for if $Q_{i}$ lay either on or above $L, P$ would lie below $L\left[a_{i}, b_{i}\right]$, violating $(\mathrm{P} 1)$ for $\left[a_{i}, b_{i}\right]$. Hence, 
when $P_{i}$ lies above $L$ for infinitely many $i, Q_{i}$ lies below $L$ for infinitely many $i$, so by the result of Case 2 (P2) holds for $[a, b]$.

Case 4: $P_{i}$ lies on $L$ for infinitely many $i$ and $Q_{i}$ lies below $L$ for only finitely many $i$. In this case choose $n$ such that $Q_{i}$ lies on or above $L$ for all $i \geq n$. $Q_{i}$ cannot lie above $L$ when $P_{i}$ lies on $L$, for if it did, then $P$ would lie below $L\left[a_{i}, b_{i}\right]$. Thus, there must be infinitely many positive integers in the set $J=\left\{j \mid j \geq n\right.$ and $P_{j}$ and $Q_{j}$ both lie on $\left.L\right\}$. Without loss of generality, we assume that $n \in J$. Since $\lim _{i \rightarrow \infty} a_{i}=a$ and $a_{i}<a$ for all $i$, there must exist some $m \in J$ such that $a_{n}<a_{m}<a$. Furthermore, it must be that $b_{m}=b$; for if $b_{m}$ were greater than $b$, there would exist $j \in J$ such that $b \leq b_{j}<b_{m} \leq b_{n}$ and $a_{n}<a_{m} \leq a_{j}<a$, and this would violate (P2) for $\left[a_{j}, b_{j}\right]$ since $(\mathrm{P} 1)$ holds for $\left[a_{n}, b_{n}\right]$. Now, $L$ contains the points $P_{m}$ and $Q_{m}$ and contains the line segment $L\left[a_{n}, b_{n}\right]$; and since (P1) holds for $\left[a_{n}, b_{n}\right]$ and $a_{m} \in\left(a_{n}, b_{n}\right), L$ is a locally supporting line for $f$ at $a_{m}$. Since (P2) holds for $\left[a_{m}, b_{m}\right], L$ must not be a locally supporting line for $f$ at $b_{m}$. But $b_{m}=b$, so we conclude that (P2) holds for $[a, b]$.

We have verified that $(\mathrm{P} 2)$ holds for $[a, b]$ in all possible situations. Since $(\mathrm{P} 1)$ and $(\mathrm{P} 2)$ both hold for $[a, b]$, we have $[a, b] \in \mathscr{F}_{f}$.

Lemma 4. Let $c \in I$, and let $\left\{\left[\dot{a}_{i}, b_{i}\right]\right\}_{i=1}^{\infty}$ be a sequence of closed subintervals of $I$, with $\left[a_{j}, b_{j}\right] \subset\left[a_{i}, b_{i}\right]$ for $j>i$ and with $\lim _{i \rightarrow \infty} a_{i}=\lim _{i \rightarrow \infty} b_{i}=c$. Suppose that $\left[a_{i}, b_{i}\right] \in \mathscr{F}_{f}$ for all $i$. Then if there exists a locally supporting line for $f$ at $c$, it is not unique.

Proof. Suppose that there does exist a locally supporting line $L$ for $f$ at $c$. Let $\varepsilon>0$ be such that for all $x \in I$ satisfying $|x-c|<\varepsilon$, the point $(x, f(x))$ lies on or above $L$; and choose $n$ such that $\left|a_{n}-c\right|<\varepsilon$ and $\left|b_{n}-c\right|<\varepsilon$. Then neither $P_{n}=\left(a_{n}, f\left(a_{n}\right)\right)$ nor $Q_{n}=\left(b_{n}, f\left(b_{n}\right)\right)$ can lie below $L$. If $P_{n}$ and $Q_{n}$ both lay on $L$, then $L$ would be a locally supporting line for $f$ at both $a_{n}$ and $b_{n}$. This cannot be, since $\left[a_{n}, b_{n}\right]$ satisfies (P2). Since $\left[a_{n}, b_{n}\right]$ also satisfies $(\mathrm{P} 1), P=(c, f(c))$ cannot lie below $L\left[a_{n}, b_{n}\right]$. This rules out having $P_{n}$ and $Q_{n}$ both lying above $L$; and if $a_{n}<c<b_{n}$, it rules out having one of the two points lying on $L$ with the other point lying above $L$. There are only two possibilities therefore: either $P_{n}=P$ and $Q_{n}$ lies above $L$ or $Q_{n}=P$ and $P_{n}$ lies above $L$. Since $\left[a_{n}, b_{n}\right]$ satisfies $(\mathrm{P} 1)$ and $L$ is a locally supporting line for $f$ at $c$, it follows that the line $L^{\prime}$ through $P_{n}$ and $Q_{n}$ is a locally supporting line for $f$ at $c$. Since $L^{\prime} \neq L, L$ is not unique.

Proof of Theorem 2. By way of contradiction, suppose that $f$ is not convex. Then either

(i) there exists an $a \in I$ such that the locally supporting line $L_{a}$ for $f$ at $a$ is not supporting, and $S=\left\{\tilde{b} \mid \tilde{b}>a\right.$ and $(\tilde{b}, f(\tilde{b}))$ lies below $\left.L_{a}\right\}$ is nonempty, or

(ii) there exists a $b \in I$ such that the locally supporting line $L_{b}$ for $f$ at $b$ is not supporting, and $T=\left\{\tilde{a} \mid \tilde{a}<b\right.$ and $(\tilde{a}, f(\tilde{a}))$ lies below $\left.L_{b}\right\}$ is nonempty. Say (i) holds, and let $b=\inf S$. Since $L_{a}$ is a locally supporting line for $f$ at $a, b>a$. Then by Lemma 1 , the point $(b, f(b))$ lies on $L_{a}$, and it follows that $[a, b] \in \mathscr{F}_{f}$. Similarly, if (ii) holds and we define $a=\sup T$, then $[a, b] \in \mathscr{F}_{f}$. In either case, $\mathscr{F}_{f}$ is nonempty. $\mathscr{F}_{f}$ is partially ordered, with the partial ordering being given by interval inclusion. Any nonempty partially ordered set contains a maximal totally ordered subset, so there exists some 
maximal subset $\mathscr{E} \subset \mathscr{F}_{f}$, totally ordered by interval inclusion.

Define $A=\{\tilde{a} \in I \mid[\tilde{a}, \tilde{b}] \in \mathscr{E}$ for some $\tilde{b} \in I\}$ and $B=\{\tilde{b} \in I \mid[\tilde{a}, \tilde{b}] \in \mathscr{E}$ for some $\tilde{a} \in I\}$. Let $\tilde{a} \in A$. Then since $\mathscr{E}$ is totally ordered by interval inclusion, $\tilde{b}>\tilde{a}$ for all $\tilde{b} \in B$. Thus, $B$ is bounded below, so $b^{*}=\inf B$ exists. Furthermore, $\tilde{a} \leq b^{*}$, and since $\tilde{a} \in A$ was arbitrary, $A$ is bounded above by $b^{*}$. Therefore $a^{*}=\sup A$ exists, and $a^{*} \leq b^{*}$.

Now we begin with any member of $\mathscr{E}$, which we designate as $\left[a_{1}, b_{1}\right]$, and define a nested sequence of intervals inductively as follows: Once $\left[a_{i}, b_{i}\right] \in \mathscr{E}$ has been defined, choose $\tilde{a}_{i+1} \in A$ such that $a^{*}-\tilde{a}_{i+1} \leq \frac{1}{2}\left(a^{*}-a_{i}\right)$. There then exists a $\tilde{b}_{i+1} \in B$ such that $\left[\tilde{a}_{i+1}, \tilde{b}_{i+1}\right] \in \mathscr{E}$. Now choose $b_{i+1} \in B$ such that $b_{i+1}-b^{*} \leq \min \left\{\tilde{b}_{i+1}-b^{*}, \frac{1}{2}\left(b_{i}-b^{*}\right)\right\}$. Since $\mathscr{E}$ is totally ordered by interval inclusion, we may now choose an $a_{i+1} \in A$ such that $a_{i+1} \geq \tilde{a}_{i+1}$ and $\left[a_{i+1}, b_{i+1}\right] \in \mathscr{E}$. This defines the interval $\left[a_{i+1}, b_{i+1}\right]$. The sequence $\left\{\left[a_{i}, b_{i}\right]\right\}_{i=1}^{\infty}$ thus produced satisfies the properties $\left[a_{i}, b_{i}\right] \in \mathscr{E}, a^{*}-a_{i} \leq$ $2^{-i+1}\left(a^{*}-a_{1}\right)$, and $b_{i}-b^{*} \leq 2^{-i+1}\left(b_{1}-b^{*}\right)$ for all $i$, so that $\lim _{i \rightarrow \infty} a_{i}=a^{*}$ and $\lim _{i \rightarrow \infty} b_{i}=b^{*}$. Furthermore, $\left[a_{j}, b_{j}\right] \subset\left[a_{i}, b_{i}\right]$ for $j>i$. It follows from Lemma 4 that $a^{*}<b^{*}$.

Now by Lemma $3,\left[a^{*}, b^{*}\right] \in \mathscr{F}_{f}$, so the line $L$ through $P=\left(a^{*}, f\left(a^{*}\right)\right)$ and $Q=\left(b^{*}, f\left(b^{*}\right)\right)$ fails to be locally supporting for $f$ at at least one of the two points $a^{*}$ and $b^{*}$, even though $(x, f(x))$ does lie on or above $L$ for $a^{*} \leq x \leq b^{*}$.

Say $L$ is not locally supporting for $f$ at $a^{*}$, and let the unique locally supporting line at $a^{*}$ be $L^{\prime}$. Then the slope of $L^{\prime}$ is greater than the slope of $L$, so $Q$ lies below $L^{\prime}$. Let $b^{\prime}=\inf \left\{\tilde{b} \mid \tilde{b}>a^{*}\right.$ and $(\tilde{b}, f(\tilde{b}))$ lies below $\left.L^{\prime}\right\}$. Then $b^{\prime}>a^{*}$ since $L^{\prime}$ is locally supporting for $f$ at $a^{*}$; and by Lemma 1 , the point $Q^{\prime}=\left(b^{\prime}, f\left(b^{\prime}\right)\right)$ lies on $L^{\prime}$. Furthermore, because of the way in which $b^{\prime}$ was chosen, $b^{\prime} \leq b^{*}$ and $L^{\prime}$ is not locally supporting for $f$ at $b^{\prime}$, although $(x, f(x))$ does lie on or above $L^{\prime}$ for $a^{*} \leq x \leq b^{\prime}$. Since $Q^{\prime} \neq Q$, we in fact have $b^{\prime}<b^{*}$. Since (P1) and (P2) now both hold for $\left[a^{*}, b^{\prime}\right]$ and $b^{\prime}<\inf B$, we have produced an interval in $\mathscr{F}_{f}$ which is a subinterval of every member of $\mathscr{E}$ but which is not itself a member of $\mathscr{E}$. This contradicts the maximality of $\mathscr{E}$.

If $L$ is not locally supporting for $f$ at $b^{*}$, a contradiction is reached in the same way. Thus, $f$ must be convex.

\section{CONVEXITY VIA LOCALlY SUPPORTING HYPERPLANES}

If $n>1$, it is possible for a function $f: \mathbb{R}^{n} \rightarrow \mathbb{R}$ to have unique local support at every point and yet not be convex. A good example of this is given by letting $f$ be the characteristic function of the set $\left\{x \in \mathbb{R}^{n} \mid\|x\|<1\right\}$ where $\|\cdot\|$ represents the usual Euclidean norm. This function is clearly not convex. To see that it does have a unique locally supporting hyperplane at every point, let $x_{0} \in \mathbb{R}^{n}$ and let $g: \mathbb{R}^{n} \rightarrow \mathbb{R}$ be affine with $g\left(x_{0}\right)=f\left(x_{0}\right)$. If $g$ is constant, then $H=\left\{(x, g(x)) \mid x \in \mathbb{R}^{n}\right\}$ is easily seen to be a locally supporting hyperplane for $f$ at $x_{0}$. If $g$ is not constant, then $H$ is not such a locally supporting hyperplane, since the open half-space $\left\{x \in \mathbb{R}^{n} \mid g(x)>f\left(x_{0}\right)\right\}$ will contain points $x$ arbitrarily close to $x_{0}$ with $\|x\|>\left\|x_{0}\right\|$. As the following theorem shows, however, local support will ensure convexity if $f$ is assumed to be continuous. 
Theorem 3. Let $S \subset \mathbb{R}^{n}$ be nonempty, open, and convex; and let $f: S \rightarrow \mathbb{R}$ be continuous. Suppose that $f$ has a locally supporting hyperplane at each $x \in S$. Then $f$ is convex.

Proof. For each pair of points $x_{1}, x_{2} \in S$ apply Theorem 1 to the function $\varphi(t)=f\left((1-t) x_{1}+t x_{2}\right)$, where $-\varepsilon<t<1+\varepsilon$, with $\varepsilon>0$ suitably small.

\section{REFERENCES}

1. N. Bourbaki, Fonctions d'une variable réelle, Hermann, Paris, 1958.

2. A. M. Bruckner, A general convexity criterion, Glas. Mat. Ser. III 13(33) (1978), 231-235.

3. A. Wayne Roberts and Dale E. Varburg, Convex functions, Academic Press, New York, 1973.

4. Clifford E. Weil, Monotonicity, convexity and symmetric derivates, Trans. Amer. Math. Soc. 221 (1976), 225-237.

5. Donald Francis Young, Local conditions for convexity and upward concavity, College Math. J. 24 (1993), 224-228.

Department of Mathematics, Southern College of Technology, Marietta, Georgia 30060

E-mail address: dyoung@st6000.sct.edu 